\title{
Generating Mutually Recursive Definitions
}

\author{
Jeremy Yallop \\ University of Cambridge, UK \\ jeremy.yallop@cl.cam.ac.uk
}

\author{
Oleg Kiselyov \\ Tohoku University, Japan \\ oleg@okmij.org
}

\begin{abstract}
Many functional programs - state machines [Krishnamurthi 2006], top-down and bottom-up parsers [Hinze and Paterson 2003; Hutton and Meijer 1996], evaluators [Abelson et al. 1984], GUI initialization graphs [Syme 2006], \&c. - are conveniently expressed as groups of mutually recursive bindings. One therefore expects program generators, such as those written in MetaOCaml, to be able to build programs with mutual recursion.

Unfortunately, currently MetaOCaml can only build recursive groups whose size is hard-coded in the generating program. The general case requires something other than quotation, and seemingly weakens static guarantees on the resulting code. We describe the challenges and propose a new language construct for assuredly generating binding groups of arbitrary size - illustrating with a collection of examples for mutual, $n$-ary, heterogeneous, value and polymorphic recursion.
\end{abstract}

CCS Concepts - Software and its engineering $\rightarrow$ Recursion; Functional languages; Source code generation;

Keywords Recursion, fixed points, multi-stage programming, metaprogramming

ACM Reference Format:

Jeremy Yallop and Oleg Kiselyov. 2019. Generating Mutually Recursive Definitions. In Proceedings of the 2019 ACM SIGPLAN Workshop on Partial Evaluation and Program Manipulation (PEPM '19), fanuary 14-15, 2019, Cascais, Portugal. ACM, New York, NY, USA, 7 pages. https://doi.org/10.1145/3294032.3294078

\section{Introduction}

MetaOCaml (whose current implementation is known as BER MetaOCaml [Kiselyov 2014]) extends OCaml with support for typed program generation. It makes three additions: $\alpha$ code is the type of unevaluated code fragments, brackets

Permission to make digital or hard copies of all or part of this work for personal or classroom use is granted without fee provided that copies are not made or distributed for profit or commercial advantage and that copies bear this notice and the full citation on the first page. Copyrights for components of this work owned by others than the author(s) must be honored. Abstracting with credit is permitted. To copy otherwise, or republish, to post on servers or to redistribute to lists, requires prior specific permission and/or a fee. Request permissions from permissions@acm.org. PEPM '19, Fanuary 14-15, 2019, Cascais, Portugal

(c) 2019 Copyright held by the owner/author(s). Publication rights licensed to ACM.

ACM ISBN 978-1-4503-6226-9/19/01 .. \$15.00

https://doi.org/10.1145/3294032.3294078 $.<\mathrm{e}>$. construct a code fragment by quoting an expression, and splices $\sim$ e insert a code fragment into a larger one.

For example, here is a function $\mathrm{t} 1$ that builds an int code fragment by inserting its int code argument within a bracketed expression:

$$
\begin{aligned}
& \text { let } \mathrm{t} 1 \mathrm{x}=.<. \sim \mathrm{X} * \text { succ } . \sim \mathrm{X}>\text {. } \\
& \sim \text { val } \mathrm{t} 1: \text { int } \operatorname{code} \rightarrow \text { int } \text { code }=<\text { fun }>
\end{aligned}
$$

and here is a call to $t 1$ with a code fragment of the appropriate type:

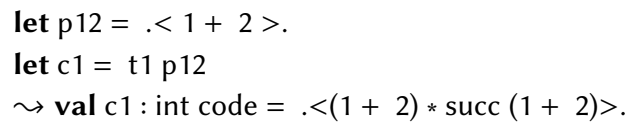

Combined with higher-order functions, effects, modules and other features of the host OCaml language, these constructs support safe and flexible program generation, permitting typed manipulation of open code while ensuring that the generated code is well-scoped and well-typed.

However, support for generating recursive programs is currently limited: there is no support for generating mutuallyrecursive definitions whose size is not hard-coded in the generating program [Taha 1999]. For example, the following state machine:

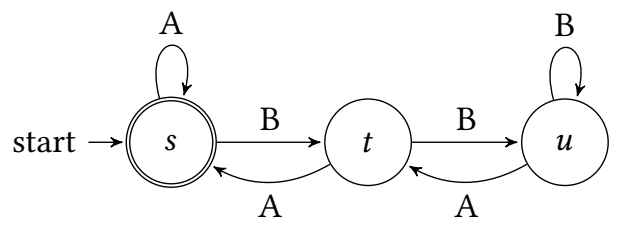

is naturally expressed as a mutually-recursive group of bindings:

$$
\begin{aligned}
\text { let rec } s & =\text { function } A:: r \rightarrow s r|B:: r \rightarrow t r|[] \\
\quad \text { and } t & =\text { function } A:: r \rightarrow s r|B:: r \rightarrow u r|[] \rightarrow \text { false } \\
\text { and } u & =\text { function } A:: r \rightarrow \operatorname{tr}|B:: r \rightarrow u r|[] \rightarrow \text { false }
\end{aligned}
$$

where each function $\mathrm{s}, \mathrm{t}$, and $\mathrm{u}$ realizes a recognizer, taking a list of A and B symbols and returning a boolean. However, the program that builds such a group from a description of an arbitrary state machine cannot be expressed in MetaOCaml.

The limited support for generating mutual recursion is a consequence of expression-based quotation: brackets enclose expressions, and splices insert expressions into expressions but a group of bindings is not an expression. There is a second difficulty: generating recursive definitions with 'backward' and 'forward' references seemingly requires unrestricted, Lisp-like gensym, which defeats MetaOCaml's static guarantees. It is unclear how to ensure all gensym-ed variables 
are eventually bound to the intended expressions, and how to ensure that generated code is well-typed.

Related metaprogramming systems such as LMS [Rompf 2016] and Template Haskell [Sheard and Jones 2002] which are capable of generating recursive definitions indeed use gensym and 'compiler magic' (such as intensional analysis of closures in LMS and dependency analysis in GHC to determine mutually recursive groups). None of them therefore catch the left-unbound variables until the code is fully generated and compiled - at which point the error in the generator becomes very difficult to find (as illustrated in [Ofenbeck et al. 2016]).

In practice, MetaOCaml programmers fall back on a variety of workarounds, simulating mutual recursion using ordinary recursion [Kiselyov 2013] or nested recursion [Inoue 2014], encoding recursion using higher-order state ("Landin's knot”) [Yallop 2016] or hard-coding templates for a few fixed numbers of binding-group sizes [Yallop 2017]. None of the workarounds are satisfactory: they do not cover all use cases, are awkward to use, or generate inefficient programs that rely on references or auxiliary data structures.

This paper solves these challenges. Specifically, it describes:

- a low-level primitive for recursive binding insertion (Section 3), building on earlier designs for insertion of ordinary let bindings (Section 2)

- a high-level combinator built on top of the low-level primitive (Section 4) that supports the generation of a wide variety of recursive patterns - mutual, $n$-ary, heterogeneous, value and polymorphic recursion.

\section{Let-Insertion}

The code generated for $\mathrm{c} 1$ above contains duplicate expressions, which ideally should be computed only once. We can avoid the duplicated computation by changing t 1 to generate a let expression:

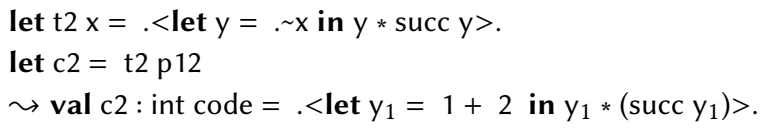

However, in general let expressions cannot be inserted locally. For example, in the following program, $\mathrm{ft} 1$ takes a code template $t$ as argument, using it when building the body of the generated function:

$$
\begin{aligned}
& \text { let } \mathrm{ft} 1 \mathrm{t} x=.<\text { fun } \mathrm{u} \rightarrow . \sim(\mathrm{t} \mathrm{x})+. \sim(\mathrm{t} .<. \sim \mathrm{x}+\mathrm{u}>\text {.) })> \\
& \sim \text { val } \mathrm{ft} 1:(\text { int code } \rightarrow \text { int code }) \rightarrow \text { int code } \rightarrow(\text { int } \rightarrow \text { int }) \text { code }
\end{aligned}
$$

Now the let expression generated by $\mathrm{t} 2$ is not positioned optimally:

$$
\begin{aligned}
& \text { let } \mathrm{c} 3=\mathrm{ft} 1 \mathrm{t} 2 \mathrm{p} 12 ; \\
& \sim \text { val } \mathrm{c} 3:(\text { int } \rightarrow \text { int }) \text { code }=.<\text { fun } \mathrm{u}_{2} \rightarrow \\
& \quad\left(\text { let } \mathrm{y}_{4}=1+2 \text { in } \mathrm{y}_{4} *\left(\operatorname{succ} \mathrm{y}_{4}\right)\right)+ \\
& \quad\left(\text { let } \mathrm{y}_{3}=(1+2)+\mathrm{u}_{2} \text { in } \mathrm{y}_{3} *\left(\text { succ } \mathrm{y}_{3}\right)\right)>.
\end{aligned}
$$

since we do not wish to compute $1+2$ every time the function generated by c3 is applied. The challenge is inserting let bindings into a wider context rather than into the immediate code fragment under construction.

Recent versions of BER MetaOCaml have a built-in genlet primitive: if e is a code value, then genlet e arranges to generate, at an appropriate place, a let expression binding e to a variable, returning the code value with just that variable. (If e is already an atomic expression, genlet e returns e as it is).

For example, in the following program $\mathrm{p} 12 \mathrm{l}$ is bound to a code expression $1+2$ that is to be let-bound according to the context. When $\mathrm{p} 12 \mathrm{l}$ is printed - that is, used in the top-level context - the let is inserted immediately:

$$
\begin{aligned}
& \text { let } \mathrm{p} 12 \mathrm{l}=\text { genlet } \mathrm{p} 12 \\
& \leadsto \text { val } \mathrm{p} 12 \mathrm{l}: \text { int code }=.<\text { let } \mathrm{I}_{5}=1+2 \text { in } \mathrm{I}_{5}>.
\end{aligned}
$$

If we pass $p 12$ to $t 1$, the let is inserted outside the template's code:

$$
\begin{aligned}
& \text { let } \mathrm{c} 1 \mathrm{l}=\mathrm{t} 1 \mathrm{p} 12 \mathrm{l} \\
& \sim \text { val } \mathrm{c} 1 \mathrm{l}: \text { int code }=.<\text { let } I_{5}=1+2 \text { in } I_{5} * \operatorname{succ} I_{5}>.
\end{aligned}
$$

Finally, in the complex $\mathrm{ft} 1$ example, the let-binding happens outside the function, as desired:

$$
\begin{aligned}
& \text { let } \mathrm{ft} 1 \mathrm{x}=.<\text { fun } \mathrm{u} \rightarrow . \sim(\mathrm{t} 1 \mathrm{x})+. \sim(\mathrm{t} 1 \text { (genlet .<. } \sim \mathrm{x}+\mathrm{u}>\text {.)) }>\text {. } \\
& \text { let } \mathrm{c} 3 \mathrm{l}=\mathrm{ft} 1 \mathrm{p} 12 \mathrm{l} ; \\
& \sim \text { val } \mathrm{c} 3 \mathrm{l}:(\text { int } \rightarrow \text { int }) \text { code }= \\
& .<\text { let } \mathrm{I}_{5}=1+2 \text { in } \\
& \quad \text { fun } \mathrm{u}_{10} \rightarrow \text { let } \mathrm{I}_{11}=\mathrm{I}_{5}+\mathrm{u}_{10} \text { in } \mathrm{I}_{5} * \text { succ } \mathrm{I}_{5}+\mathrm{I}_{11} * \text { succ } \mathrm{I}_{11}>\text {. }
\end{aligned}
$$

Let-insertion and memoization Let-insertion is often used with memoization, as we illustrate with a simplified dynamic-programming algorithm [Kameyama et al. 2011]. The fibnr function computes the nth element of the Fibonacci sequence whose first two elements are given as arguments $\mathrm{x}$ and y:

$$
\begin{aligned}
& \text { let fibnr plus } x \text { y self } n= \\
& \text { if } n=0 \text { then } x \text { else } \\
& \text { if } n=1 \text { then } y \text { else } \\
& \text { plus }(\operatorname{self}(n-1))(\text { self }(n-2))
\end{aligned}
$$

The code is written in open-recursive style, and abstracted over the addition operation. Tying the knot with the standard call-by-value fixpoint combinator let rec fix $f x=f($ fix $f) x$ we compute, for example, the 5 th element of the standard sequence as fix (fibnr (+) 11 1) 5 .

If, instead of passing the standard addition function + for fibnr's plus argument, we pass a code-generating implementation of plus then fibnr also becomes a code generator, here building code that computes the 5 th element, given the first two:

$$
\begin{aligned}
& \text { let splus } x y=.<. \sim x+. \sim y>\text {. in } \\
& .<\text { fun } x y \rightarrow . \sim(\text { fix }(\text { fibnr splus } .<x>. .<y>\text {.) } 5)>\text {. } \\
& \leadsto-:(\text { int } \rightarrow \text { int } \rightarrow \text { int }) \text { code }= \\
& .<\text { fun } x_{1} y_{2} \rightarrow\left(\left(\left(y_{2}+x_{1}\right)+y_{2}\right)+\left(y_{2}+x_{1}\right)\right)+\left(\left(y_{2}+x_{1}\right)+y_{2}\right)>\text {. }
\end{aligned}
$$


The duplicated expressions in the generated code reveal why fibnr is exponentially slow.

A memoizing fixpoint combinator inserts a let-binding for the result of each call, and maintains a mapping from previous arguments to the let-bound variables [Swadi et al. 2006]

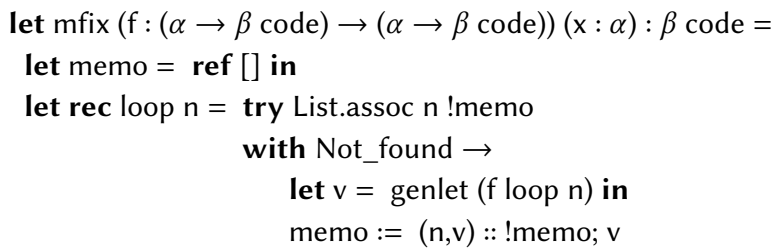

in loop $\mathrm{x}$

letting us compute the n-th element fast and generate fast code:

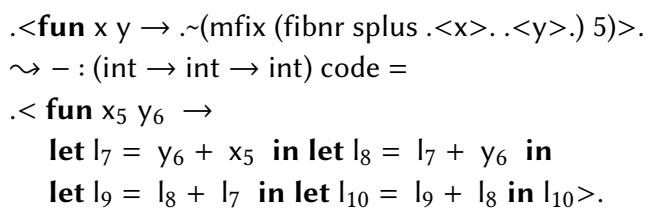

Without genlet however, we get the same poor code as with the ordinary fix: memoization alone speeds up the code generation without affecting the efficiency of the generated code. The crucial role of let-insertion in these applications has been extensively discussed by Swadi et al. [2006].

\section{Inserting Recursive Let}

As we have seen, the specialization of recursive functions calls for generating definitions. More complicated recursive patterns require generating recursive definitions. The simplest example is specializing the Ackermann function

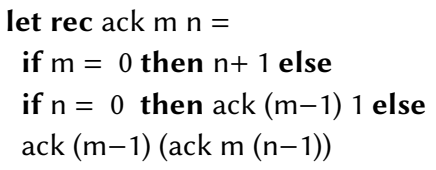

for a given value of $\mathrm{m}$, a challenge originally posed by Neil Jones. Turning ack into a generator of specialized code is easy in the open-recursion style, by merely annotating the code keeping in mind that $\mathrm{n}$ is future-stage:

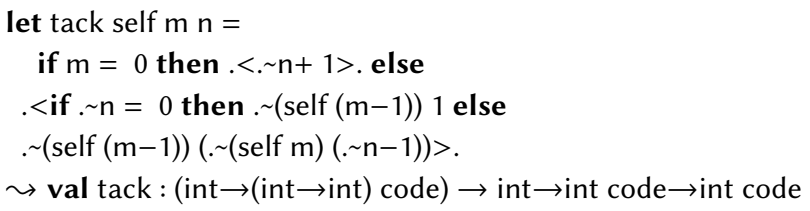

All that is left is to set the desired value of $m$ and apply the mfix - which promptly diverges:

mfix (fun self $\mathrm{m} \rightarrow .<$ fun $\mathrm{n} \rightarrow . \sim($ tack self $\mathrm{m} .<\mathrm{n}>$.) $>$.) 2

Looking at the original ack shows the reason: ack $m$ depends not only on ack $(\mathrm{m}-1)$ but also on ack $\mathrm{m}$ itself.
Generating recursive definitions was deemed for a long time a difficult problem. One day, a two-liner solution emerged, from the insight that a recursive definition

$$
\text { let rec } g=\text { in body }
$$

may be re-written as

$$
\text { let } \mathrm{g}=\text { let rec } \mathrm{g}=\mathrm{e} \text { in } \mathrm{g} \text { in body }
$$

which immediately gives us genletrec:

$$
\begin{aligned}
& \text { let genletrec }:((\alpha \rightarrow \beta) \text { code } \rightarrow \alpha \text { code } \rightarrow \beta \text { code }) \rightarrow \\
& \qquad(\alpha \rightarrow \beta) \text { code }= \\
& \text { fun } \mathrm{f} \rightarrow \text { genlet } .<\text { let rec } \mathrm{g} \mathrm{x}=. \sim(\mathrm{f} .<\mathrm{g}>\ldots \mathrm{x}>\text {. }) \text { in } \mathrm{g}>.
\end{aligned}
$$

The new memoizing fixpoint combinator becomes

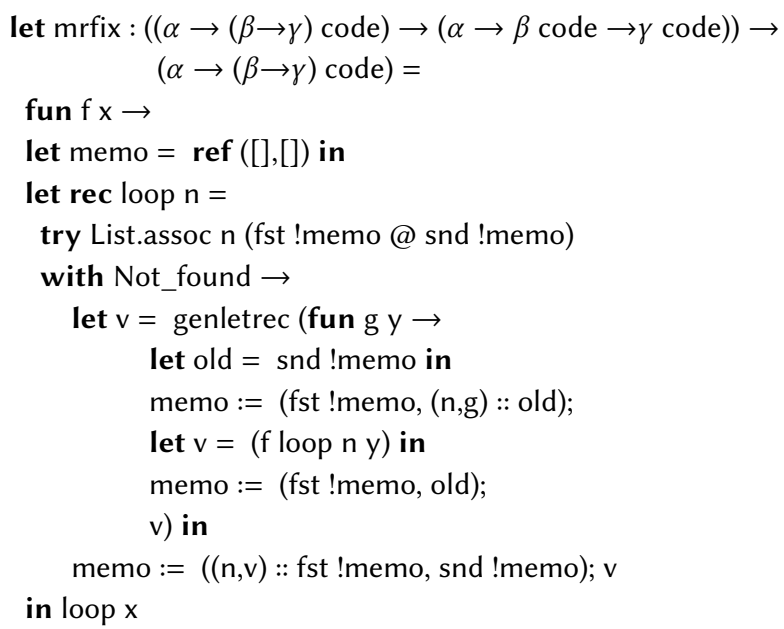

Recursive definitions have to be the definitions of functions: this fact is reflected in mrfix's (and genletrec's) code and type. The mrfix code has another peculiarity: splitting of the memo table into the 'global' and 'local' parts. We let the reader contemplate its significance (until we return to this point in Section 4).

Finally we are able to specialize the Ackermann function to a particular value of $\mathrm{m}$ (which is two, in the code below):

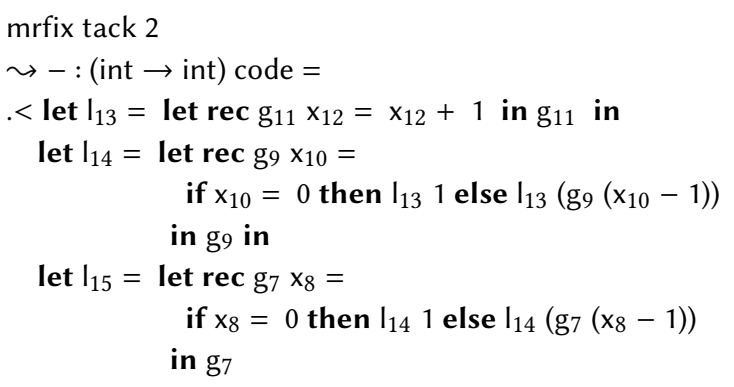

in $\mathrm{I}_{15}>$.

One clearly sees recursive definitions that were not present in the original ack. 


\section{Generating Mutually-Recursive Functions}

In many practical cases of generating recursive definitions one wants to produce mutually recursive definitions, such as the state machine shown in Section 1. To illustrate the challenges brought by mutual recursion, we take a simpler running example, contrived to be in the shape of the earlier Ackermann function. The example is the 'classical' evenodd pair, but taking two non-negative integers $m$ and $n$ and returning a boolean, telling if the sum $m+n$ has even or odd parity, resp.

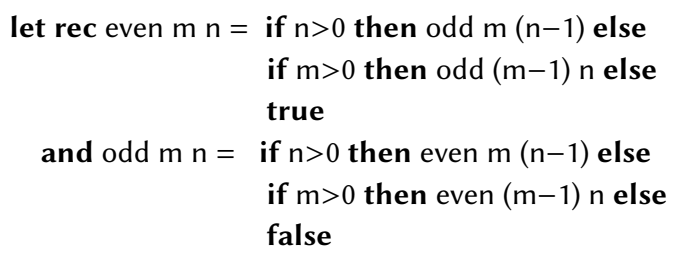

At first, mutual recursion seems to pose no problem: after all, a group of mutually recursive functions may always be converted to the ordinary recursive function by adding an extra argument: the index of a particular recursive clause in the group ${ }^{1}$ :

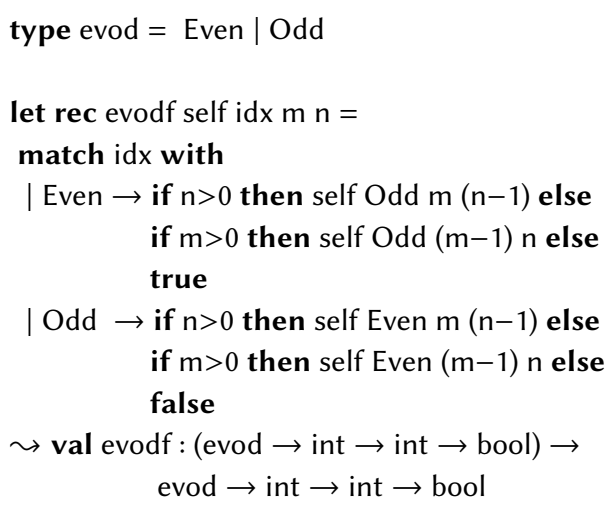

To find out if the sum of 10 and 42 has even parity one writes fix evodf Even 10+42. The straightforward staging gives

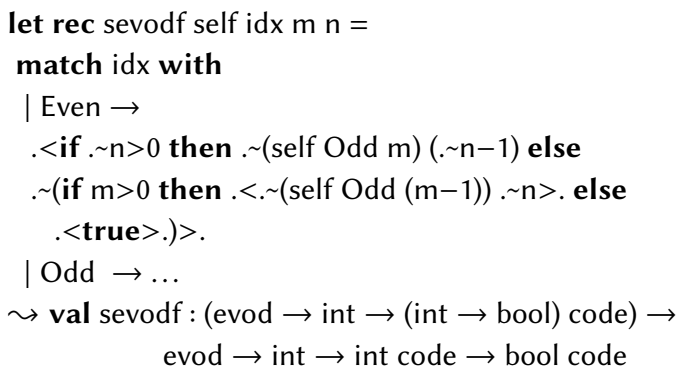

which looks very much like tack from Section 3. We could thus apply mrfix from that section with trivial adaptations

\footnotetext{
${ }^{1}$ Since the functions even and odd have the same types, the index here is the ordinary data type evod. The general case calls for generalized algebraic data types (GADTs), as Section 5.3 shows.
}

and obtain the code for even $\mathrm{m} \mathrm{n}$ specialized to a particular value of $\mathrm{m}$, say, 0 (which is just the ordinary even function):

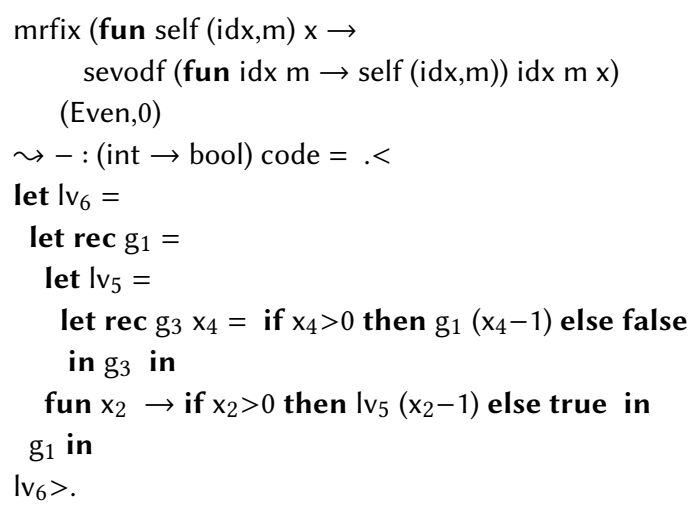

The odd function (appearing under the generated name $g_{3}$ ) is nested inside even (or, $\mathrm{g}_{1}$ ) rather than being 'parallel' with it. It means odd is not accessible from the outside; if we also want to compute odd parity, we have to duplicate the code. There is a deeper problem than mere code duplication: specializing even $\mathrm{m} n$ to $\mathrm{m}=1$ (that is, applying the tied-knot sevodf to (Even,1)) generates no code. An exception is raised instead, telling us that MetaOCaml detected scope extrusion: an attempt to use a variable outside the scope of its binding. Indeed, we have attempted to produce something like the following (identifiers are renamed for clarity):

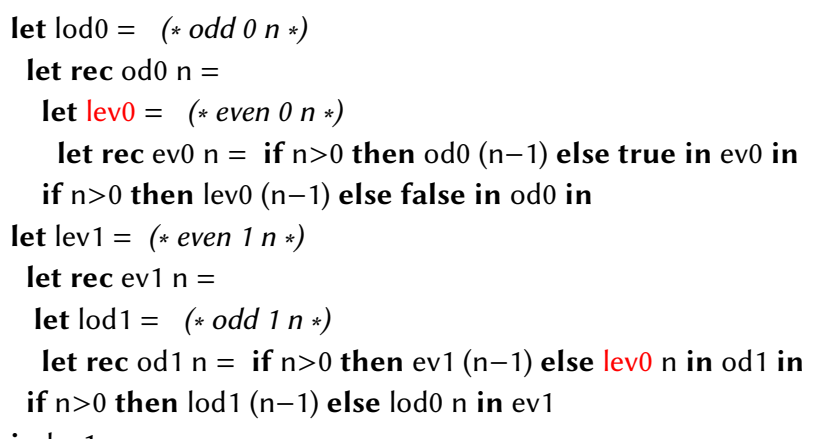

Here, the function ev1, the specialization of even $\mathrm{m} \mathrm{n}$ to $m=1$ calls od 0 and od1. The latter calls ev1 and fun $\mathrm{n} \rightarrow$ even $0 \mathrm{n}$, whose code was already generated and memoized, under the name lev0. Unfortunately, the scope of lev 0 does not extend beyond the scope of od0's definition, and hence mentioning lev 0 within od 1 is a scope extrusion.

We would like to generate the mutually recursive definition let rec even $=\ldots$ and odd $=\ldots$ that defines both even and odd in the same scope. Alas, this is impossible using only brackets and escapes: code values represent OCaml expressions, but the set of bindings is not an expression. There is also a bigger, semantic challenge. While generating the code for the i-th recursive clause in a group we may refer to clauses with both smaller and larger indices. It seems we have to resort to Lisp-like gensym, explicitly creating a name and only later binding it. However, what static assurances 
do we have that all generated names will be bound, and to their intended clauses? How do we maintain the MetaOCaml guarantee that the fully generated code is always well-typed?

Finally, what should the interface for the generator of mutually recursive bindings be in the first place? After quite a bit of thought, it turns out that genletrec's interface would suffice. For the sake of better error detection, one would generalize it slightly. We add a second function, genletrec_locus, which marks the location where a group of recursive definitions should be inserted; the generated locus_t value representing the location can be passed as first argument of genletrec:

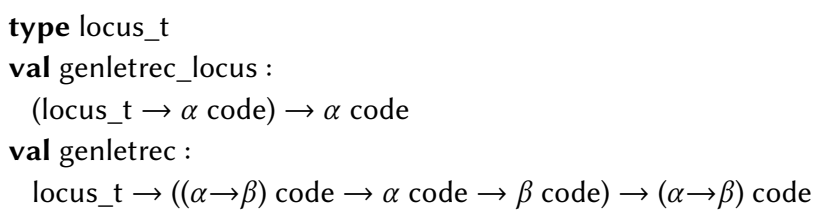

The earlier genlet (and, hence genletrec) inserted the requested definition in the widest possible context (while ensuring the absence of unbound variables in the generated code). With the new interface the insertion point (and hence the scope of the inserted bindings) is explicitly marked using genletrec_locus and each call to genletrec indicates which group of recursive bindings should contain the generated definition $^{2}$. Correspondingly, in a call

genletrec locus (fun $\mathrm{g} x \rightarrow \ldots$ )

the identifier for the binding (bound to g) scopes beyond genletrec's body (but within the scope denoted by locus).

The new genletrec let us write mrfix essentially just like the simpler mfix, without the splitting of the memo table into global and local parts ${ }^{3}$ : now, the definitions have the same scope.

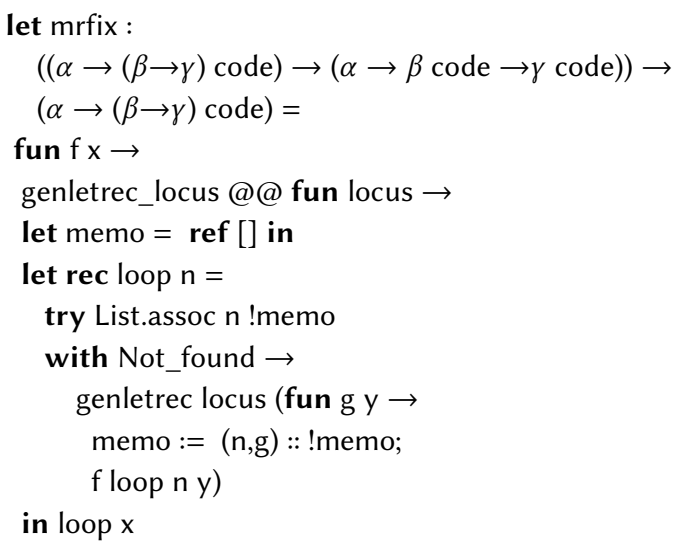

\footnotetext{
${ }^{2}$ It hence becomes the programmer's responsibility to place genletrec_locus correctly. We are yet to explore and resolve the trade-off between automatically floating genlet and genletrec whose scope is to be set manually.

${ }^{3}$ Previously, genletrec relied on the trick let $\mathrm{g}=$ let rec $\mathrm{g}=\mathrm{e}$ in $\mathrm{g}$ in body, which binds two different gs, one within and one outside the scope of the local let rec. Therefore, the memo table had two parts. The local part tracks the identifiers that are valid only while we are generating the let rec body; the global part, to which we only add, collects the externally visible gs.
}

With this new mrfix but the same sevodf from Section 4 we are able to generate the specialized even $1 \mathrm{n}$ code, with four mutually recursive definitions.

Finite State Automata, reprise Recognizers of finite state automata are produced by the following generic, textbook generator $^{4}$ :

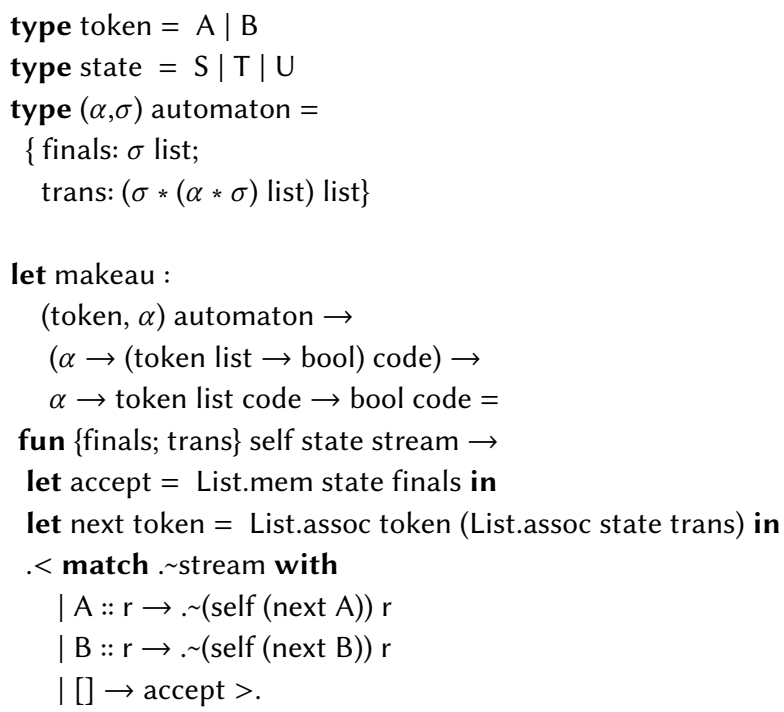

In particular, the automaton in Section 1 is represented by the following description

$$
\begin{aligned}
\text { let au } 1= & \\
\{\text { finals }= & {[\mathrm{S}] ; } \\
\text { trans }= & {[(\mathrm{S},[(\mathrm{A}, \mathrm{S}) ;(\mathrm{B}, \mathrm{T})]) ;} \\
& (\mathrm{T},[(\mathrm{A}, \mathrm{S}) ;(\mathrm{B}, \mathrm{U})]) ; \\
& (\mathrm{U},[(\mathrm{A}, \mathrm{T}) ;(\mathrm{B}, \mathrm{U})]) ;]\}
\end{aligned}
$$

Then mrfix (makeau au1) S generates:

$$
\begin{aligned}
\text { let rec } x_{1} y= & \text { match } y \text { with } \\
& \mid A:: r \rightarrow x_{1} r \\
& \mid B:: r \rightarrow x_{5} r \\
& \mid[] \rightarrow \text { true } \\
\text { and } x_{5} y= & \text { match } y \text { with } \\
& \mid A:: r \rightarrow x_{1} r \\
& \mid B:: r \rightarrow x_{9} r \\
& \mid[] \rightarrow \text { false } \\
\text { and } x_{9} y= & \text { match y with } \\
& \mid A:: r \rightarrow x_{5} r \\
& \mid B:: r \rightarrow x_{9} r \\
& \mid[] \rightarrow \text { false }
\end{aligned}
$$

in $\mathrm{x}_{1}$

of the type token list $\rightarrow$ bool.

\footnotetext{
${ }^{4}$ The generator makeau is indeed polymorphic over the type of the state; the dependence on the alphabet shows in the match statement. Incidentally, MetaOCaml also has a facility to generate pattern-match clauses of statically unknown length and content. With its help, we can make makeau fully general.
} 


\section{Further Extensions}

We sketch some extensions to the mrfix combinator of Section 4 .

\subsection{Arbitrary Bodies in let rec Expressions}

The mrfix combinator has the following type:

$$
\begin{gathered}
\text { val mrfix }:((\alpha \rightarrow(\beta \rightarrow \gamma) \text { code }) \rightarrow(\alpha \rightarrow \beta \text { code } \rightarrow \gamma \text { code })) \rightarrow \\
\qquad \alpha \rightarrow(\beta \rightarrow \gamma) \text { code }
\end{gathered}
$$

There are two arguments: the first is a function that builds recursive definitions; the second (of type $\alpha$ ) is an index that selects the identifier associated with one of the definitions to appear in the body of the generated let rec expression. For example, in the code generated for the Ackermann function by the call mrfix tack 2 in Section 3, the body of the generated expression is $I_{15}$, the identifier associated with the definition generated by tack 2 . And in the code generated for the finite state automaton in Section 4 the body of the generated expression is $x_{1}$, the name of the function that corresponds to the start symbol.

However, it is sometimes convenient to generate let rec expressions with bodies that are more complex than single identifiers. The following function, mrfixk, generalizes mrfix to additionally support generation of arbitrary bodies:

$$
\begin{gathered}
\text { val mrfixk }:((\alpha \rightarrow(\beta \rightarrow \gamma) \text { code }) \rightarrow(\alpha \rightarrow \beta \text { code } \rightarrow \gamma \text { code })) \rightarrow \\
((\alpha \rightarrow(\beta \rightarrow \gamma) \text { code }) \rightarrow \gamma \text { code }) \rightarrow \gamma \text { code }
\end{gathered}
$$

Rather than an index, the second argument is now a function that calls its argument to insert recursive definitions and builds a body of type $\gamma$ code. For example, here is the code that builds a recursive group representing the state machine from previous examples, whose body is a tuple returning all the recognizer functions:

$$
\text { mrfixk (makeau au1) (fun } \mathrm{f} \rightarrow .<(. \sim(\mathrm{f} \mathrm{S}), . \sim(\mathrm{f} U), . \sim(\mathrm{f} \mathrm{T}))>\text {.) }
$$

The generated code is the same as the code generated by mrfix, except for the more complex body:

$$
\begin{array}{r}
\text { let rec } x_{1} y=\text { match } y \text { with } A:: r \rightarrow x_{1} r \\
\mid B:: r \rightarrow x_{5} r \\
\mid[] \rightarrow \text { true } \\
\text { and } x_{5} y=\text { match } y \text { with } A:: r \rightarrow x_{1} r \\
\mid B:: r \rightarrow x_{9} r \\
\mid[] \rightarrow \text { false } \\
\text { and } x_{9} y=\text { match } y \text { with } A:: r \rightarrow x_{5} r \\
\mid B:: r \rightarrow x_{9} r \\
\mid[] \rightarrow \text { false }
\end{array}
$$

in $\left(x_{1}, x_{9}, x_{5}\right)$

\subsection{A Syntax Extension}

Third-order functions such as mrfixk are not always easy to understand and use. The following small syntax extension improves readability in many cases:

$$
\begin{aligned}
& \text { let\%staged rec } f p p^{\prime}=\text { e in } e^{\prime} \\
& \sim \operatorname{mrfixk}\left(\text { fun } f p p^{\prime} \rightarrow \text { e) }\left(\text { fun } f \rightarrow e^{\prime}\right)\right.
\end{aligned}
$$

Here \%staged is an attribute that indicates the need for a rewrite by a plug-in program that expands the syntax as shown above.

Then ack can be written as follows

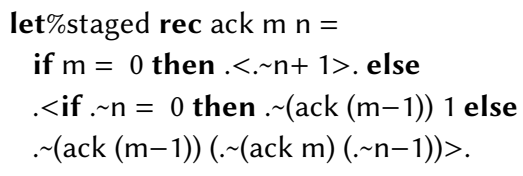

$$
\text { in ack } 2
$$

As this example shows, the syntax extension avoids the need for explicitly higher-order code and for open recursion; the identifier ack serves as the self argument in the expanded syntax, and so the calls to ack appear as standard recursion.

\subsection{Heterogeneously-Typed Recursive Groups}

In the examples up to this point the bindings in each recursive group have all been of a single type. In practice, however, it is common for let rec to bind definitions of different types. Supporting this general case requires several changes to the type of the fixpoint combinator to make it more polymorphic.

The central idea is to generalize the index types used to select recursive bindings from regular algebraic data types to GADTs. ${ }^{5}$ For example, the following GADT supports generating mutually-recursive bindings for functions of types int $\rightarrow$ bool and float $\rightarrow$ bool

$$
\begin{aligned}
\text { type } \alpha \text { eo }= & \text { Even }:(\text { int } \rightarrow \text { bool }) \text { eo } \\
& \mid \text { Odd }:(\text { float } \rightarrow \text { bool }) \text { eo }
\end{aligned}
$$

The type of the mrfixk function is generalized accordingly: the type $\alpha \rightarrow(\beta \rightarrow \gamma)$ code of functions that map indexes to variables becomes $\forall \alpha . \alpha \tau \rightarrow \alpha$ code, where the higherkinded type variable $\tau$ stands for an arbitrary parameterized index type, such as eo. Here is the fully generalized type:

$$
\begin{array}{r}
\text { val mrfixk : } \forall \gamma \forall \tau .(\forall \beta .(\forall \alpha . \alpha \tau \rightarrow \alpha \text { code }) \rightarrow(\beta \tau \rightarrow \beta \text { code })) \rightarrow \\
((\forall \alpha . \alpha \tau \rightarrow \alpha \text { code }) \rightarrow \gamma \text { code }) \rightarrow \gamma \text { code }
\end{array}
$$

Since the higher-rank and higher-kinded polymorphism found in this type cannot be expressed directly in OCaml, our implementation uses standard encodings based on OCaml's polymorphic record fields and functors.

\subsection{Polymorphic Recursion}

The extensions needed to support heterogeneous recursion (Section 5.3) are also sufficient to support polymorphic recursion. For example, here is a nested data type ntree of perfectly balanced trees and a polymorphic function swivel that interchanges left and right elements of ntree values:

\footnotetext{
${ }^{5}$ This (progressively fancier and fancier) indexing is closely related to the generalized arity in Plotkin and Power's formulation of algebraic effects [Plotkin and Power 2003]. Like them, we represent a tuple as a set of indices plus the function that maps each index to a value. The index set does not have to have a fixed finite cardinality. Adding more structure to the set of indices lets us, like it let Plotkin and Power, represent more interesting collections.
} 


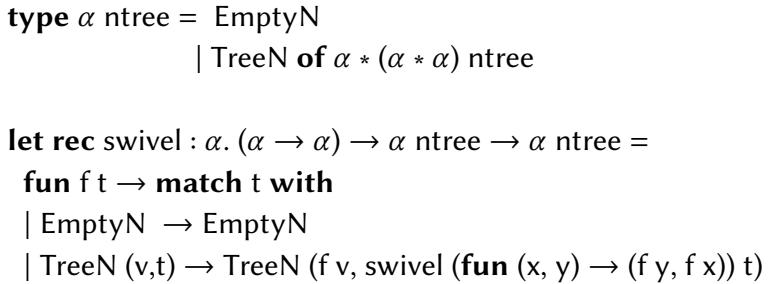

This is polymorphic-recursive because the recursive call uses swivel at a different type than the type of the definition: the passed function $\mathrm{f}$ acts on pairs $\alpha * \alpha$, not values of type $\alpha$.

Generating polymorphic-recursive definitions like swivel involves indexing by a polymorphic type. Here is a suitable index for generating swivel:

type swivel $=\{$ swivel: $\alpha .(\alpha \rightarrow \alpha) \rightarrow \alpha$ ntree $\rightarrow \alpha$ ntree $\}$

type _ index $=$ Swivel : swivel index

At each use of the index the polymorphic record field can be instantiated afresh, making it possible to call the generated function recursively at any instance of the type $(\alpha \rightarrow \alpha) \rightarrow \alpha$ ntree $\rightarrow \alpha$ ntree.

\section{Acknowledgments}

We thank Nada Amin and Jun Inoue for helpful discussions and posed challenges, and Atsushi Igarashi for hospitality. We are grateful to anonymous reviewers for many helpful suggestions. This work was partially supported by JSPS KAKENHI Grant Number 18H03218.

\section{Status}

The described genletrec is prototyped ${ }^{6}$ in full using plain MetaOCaml as well as MetaOCaml with delimited control effects, such as those provided by Multicore OCaml [Dolan et al. 2015] or the delimcc library [Kiselyov 2012]. We are working at supporting it above-the-board in a forthcoming release of MetaOCaml.

\section{References}

Hal Abelson, Jerry Sussman, and Julie Sussman. 1984. Structure and Interpretation of Computer Programs. MIT Press. ISBN 0-262-01077-1.

Stephen Dolan, Leo White, KC Sivaramakrishnan, Jeremy Yallop, and Anil Madhavapeddy. 2015. Effective Concurrency through Algebraic Effects. (September 2015). OCaml Users and Developers Workshop 2015.

Ralf Hinze and Ross Paterson. 2003. Derivation of a Typed Functional LR Parser.

${ }^{6}$ https://github.com/yallop/metaocaml-letrec
Graham Hutton and Erik Meijer. 1996. Monadic Parser Combinators. Technical Report NOTTCS-TR-96-4. Department of Computer Science, University of Nottingham.

Jun Inoue. 2014. Supercompiling with Staging. In Fourth International Valentin Turchin Workshop on Metacomputation.

Yukiyoshi Kameyama, Oleg Kiselyov, and Chung-chieh Shan. 2011. Shifting the Stage: Staging with Delimited Control. f. Funct. Program. 21, 6 (Nov. 2011), 617-662.

Oleg Kiselyov. 2012. Delimited Control in OCaml, Abstractly and Concretely. Theor. Comput. Sci. 435 (June 2012), 56-76. https://doi.org/10.1016/j.tcs. 2012.02.025

Oleg Kiselyov. 2013. Simplest poly-variadic fix-point combinators for mutual recursion. http://okmij.org/ftp/Computation/fixed-point-combinators. html\#Poly-variadic.

Oleg Kiselyov. 2014. The Design and Implementation of BER MetaOCaml. In Functional and Logic Programming (Lecture Notes in Computer Science), Michael Codish and Eijiro Sumii (Eds.), Vol. 8475. Springer International Publishing, 86-102.

Shriram Krishnamurthi. 2006. Educational Pearl: Automata via macros. f. Funct. Program. 16, 3 (2006), 253-267. https://doi.org/10.1017/ S0956796805005733

Georg Ofenbeck, Tiark Rompf, and Markus Püschel. 2016. RandIR: differential testing for embedded compilers. In Proceedings of the 7th ACM SIGPLAN Symposium on Scala, SCALA@SPLASH 2016. ACM, 21-30. https://doi.org/10.1145/2998392

Gordon Plotkin and John Power. 2003. Algebraic Operations and Generic Effects. Applied Categorical Structures 11, 1 (01 Feb 2003), 69-94. https: //doi.org/10.1023/A:1023064908962

Tiark Rompf. 2016. The Essence of Multi-stage Evaluation in LMS. Springer International Publishing, Cham, 318-335. https://doi.org/10.1007/ 978-3-319-30936-1_17

Tim Sheard and Simon Peyton Jones. 2002. Template Meta-programming for Haskell. SIGPLAN Not. 37, 12 (Dec. 2002), 60-75. https://doi.org/10. $1145 / 636517.636528$

Kedar Swadi, Walid Taha, Oleg Kiselyov, and Emir Pašalić. 2006. A Monadic Approach for Avoiding Code Duplication When Staging Memoized Functions. In PEPM. 160-169.

Don Syme. 2006. Initializing Mutually Referential Abstract Objects: The Value Recursion Challenge, In Proceedings of the ACM-SIGPLAN Workshop on ML (2005).

Walid Mohamed Taha. 1999. Multistage Programming: Its Theory and Applications. Ph.D. Dissertation. Oregon Graduate Institute of Science and Technology. AAI9949870.

Jeremy Yallop. 2016. Staging Generic Programming. In Proceedings of the 2016 ACM SIGPLAN Workshop on Partial Evaluation and Program Manipulation (PEPM '16). ACM, New York, NY, USA, 85-96. https: //doi.org/10.1145/2847538.2847546

Jeremy Yallop. 2017. Staged Generic Programming. Proc. ACM Program. Lang. 1, ICFP, Article 29 (Aug. 2017), 29:1-29:29 pages. https://doi.org/ $10.1145 / 3110273$ 